12

\title{
Диагностика пучков заряженных частиц методом фазовых диаграмм
}

\author{
(C) В.В. Лукашевич \\ Научно-исследовательский центр „Курчатовский институт“ - ПИЯФ им. Б.П. Константинова, \\ 188300 Гатчина, Ленинградская обл., Россия \\ e-mail: lukashevich_vv@pnpi.nrcki.ru
}

Поступило в Редакцию 11 марта 2019 г.

В окончательной редакции 19 сентября 2019 г.

Принято к публикации 19 сентября 2019 г.

Для определения качества фокусировки заряженных частиц в ионно-оптических устройствах предложено использовать метод фазовых диаграмм. Фазовые диаграммы с нелинейными контурами указывают на нелинейность фокусировки пучка частиц, причем наглядно наблюдается величина апертуры, при которой появляется и усиливается нелинейность. Это открывает возможность проектирования линейно фокусирующих линз или корректоров аберраций, компенсирующих нелинейности оптики.

Ключевые слова: ионная оптика, эмиттанс, линзы, фазовая диаграмма, аберрации.

DOI: $10.21883 / J T F .2020 .03 .48934 .90-19$

\section{Введение}

Разрешающая способность масс-сепараторов или спектрометров обратно пропорциональна угловому разбросу пучка частиц, формируемого электростатическими линзами. Для параксиальных пучков, когда справедливо линейное приближение, угловой разброс уменьшается с ростом ширины, и разрешающая способность растет пропорционально. Эта пропорциональность нарушается с появлением аберраций. Для анализа и контроля аберрационных вкладов необходим метод или инструмент для их наблюдения.

В традиционном подходе в оптике заряженных частиц находятся соотношения между параметрами траектории в плоскости наблюдения после прохождения частицей системы электрических и магнитных полей и начальными параметрами, определяющими траекторию перед входом частицы в область поля.

Эти соотношения являются решениями дифференциальных уравнений движения и представляются в виде ряда по степеням начальных параметров. Этот ряд, напоминающий ряд Тейлора, имеет следующий вид:

$x_{a}^{f}=\sum L_{a b} x_{b}^{i}+\sum M_{a b c} x_{c}^{i} x_{b}^{i}+\sum N_{a b c d} x_{d}^{i} x_{c}^{i} x_{b}^{i}+\ldots$.

Первый член в этом выражении определяет линейное приближение, а последующие члены, более высокого порядка, интерпретируются как аберрации. Аберрационные поправки в силу их малости находятся из решений неоднородных уравнений движения методом теории возмущений.

Классификация аберрационных коэффициентов и их вычисление для некоторых оптических элементов, таких как аксиально симметричные, квадрупольные и трансаксиальные линзы, секторные магниты и т.д. посвящено огромное количество публикаций. Ссылки на подобные работы представлены во многих монографиях, напри- мер [1-3], и регулярно издаваемых трудах EMIS (электромагнитные изотоп-сепараторы) конференций.

В настоящей работе для анализа оптических свойств электромагнитных фокусирующих систем используется эмпирический подход, позволяющий наглядно наблюдать интегральную нелинейность преобразования траекторий частиц. Этот подход основан на методе фазовых диаграмм пучка. Суть метода заключается в следующем. Представим себе отрезок прямой линии, заселенный фазовыми точками. Тогда при линейных преобразованиях этот отрезок может изменить длину и ориентацию, но останется отрезком прямой линии. Это утверждение можно изложить в другой форме. Частицы, вылетающие из точки объекта, собираются в соответствующую точку изображения. Когда линейность нарушена, указанный отрезок искривляется. Точка в изображении в этом случае становится пятном.

Линейность (или нелинейность) преобразования траекторий ионов любым ионно-оптическим элементом и системой в целом оценивается по виду фазовых диаграмм, измеренных в интересуемых сечениях пучка ионов. На основе таких измерений становится возможным проектирование и оптимальная настройка линейных (при отсутствии аберраций) фокусирующих систем. Заметим, что такой подход, использующий анализ фазовых диаграмм, используется впервые.

Конечной целью развитого формализма является создание линейно-фокусирующих систем с последующим их использованием в масс-сепараторе, разрешающая способность которого ограничена только фазовым объемом пучка и его шириной.

Масс-сепаратор с линейно фокусирующей системой предполагается использовать на нейтронном канале реактора ПИК или циклотрона ОФВЭ Петербургского института ядерной физики в рамках проекта „ИРИНА“ [4]. Все новые результаты получены на основе компьютерного моделирования движения ионов в электрических и магнитных полях. 


\section{1. Фазовая диаграмма}

Понятия фазового пространства, фазовой диаграммы и эмиттанса как площади этой диаграммы следует из теоремы Лиувилля классической механики. Фазовый объем - это шестимерный интеграл по трем проекциям координат и трем компонентам импульсов частиц. Теорема Лиувилля гласит, что при отсутствии диссипативных сил, исчезновения или рождения частиц, фазовый объем сохраняется, хотя форма его может изменяться. В этом смысле фазовый объем похож на пузырь с несжимаемой жидкостью. Его можно деформировать, но объем остается тем же.

Исторически фазовые диаграммы были введены при развитии ускорительной техники. Они с успехом применяются для исследования фазовых колебаний, особенно при расчете условий захвата частиц в процессе ускорения в синхротронах и накопления ускоренных пучков в накопительных кольцах. Важную роль они играют при конструировании систем транспортировки пучков ускорителей и ионных пучков сепараторов. Теорема Лиувилля применяется во многих других областях физики, например, в физике плазмы при изучении плазменных колебаний, а также при описании эволюции звездных скоплений, в которых движение звезд происходит в собственных гравитационных полях.

Интересно отметить как формальную, так и физическую аналогию между эмиттансом и энтропией. Оба представления отражают меру случайности и отсутствие упорядоченности в распределении фазовых точек в пределах диаграммы. Связь между эмиттансом $\varepsilon$ и энтропией $S$ установлена в работе [5]: $S=k N(\lg \pi \varepsilon-\lg \delta)$. Здесь $k$ - постоянная Больцмана, $N$ - число частиц, $\delta$ - размер фазовой ячейки. Если пучок фокусируется для получения изображения или массовой линии в фокальной плоскости сепаратора или спектрометра, то наличие аберраций приводит к потере информации, так как разрешающая способность уменьшается. Фазовая точка имеет размерность действия и в квантовом пределе размер фазовой ячейки определяется постоянной Планка. В классическом случае этот размер зависит от точности измерений, а при вычислениях от точности расчета. Точность вычислений, в свою очередь, определяется размером элементарной ячейки объемной сетки, на которой проводятся вычисления.

Заметим, что экспериментально измерить фазовую диаграмму чрезвычайно сложно. Это невозможно сделать внутри электростатических фокусирующих систем, где имеются электрические поля, так как измерительные устройства искажают распределение полей. Пучки частиц высоких энергий имеют большую проникающую способность, и щели измерительной системы должны иметь массивные блоки, не пропускающие частицы. Это также затрудняет измерение фазовых диаграмм и эмиттансов ускоренных пучков.

В ускорительной технике благодаря фазовым (синхротронным) колебаниям частиц фазовые диаграммы пуч- ков представляются в виде эллипсов. В ионной оптике характер движения частиц другой и фазовые диаграммы при отсутствии аберраций имеют линейные контуры в вид ромбов или параллелограммов.

Поскольку фазовая диаграмма пучка является инструментом контроля качества фокусировки, кратко опишем ее свойства. Шестимерный интеграл фазового объема сокращается до четырехмерного, если свойства пучков рассматриваются в каких-то сечениях и продольные скорости во много раз больше поперечных. Далее в линейном приближении уравнения движения по вертикальным и горизонтальным переменным разделяются, и траектории в вертикальной и горизонтальных плоскостях становятся независимыми. В этом случае четырехмерный интеграл сводится к двум двумерным интегралам, каждый из которых представляет собой площадь фазовой диаграммы в координатах „угол наклона-отклонение от оси“. Для примера рассмотрим вертикальную компоненту импульса:

$$
d p_{y}=m d\left(\frac{d y}{d x} \frac{d x}{d t}\right)=m d y^{\prime} v_{x} \simeq \sqrt{2 m e \varphi} d y^{\prime}
$$

Для частиц с определенной массой и заданной величиной энергии компонента скорости выражается через наклон траектории $y^{\prime}$, и фазовая диаграмма имеет вышеуказанные координаты $y^{\prime}-y$. В этих координатах каждая частица отображается в виде фазовой точки, и совокупность этих точек заполняет некоторую область, представляющую собой фазовую диаграмму пучка частиц. Для расходящегося пучка фазовая диаграмма ориентирована в первом и третьем квадрантах, а для сходящегося - во втором и четвертом. Сечение пучка, диаграмма которого ориентирована вдоль одной из осей координат, будем называть кроссовером. Существует два типа кроссоверов. Кроссовер широкого пучка с малыми углами наклона траекторий. Это пучок с приблизительно параллельными траекториями. В этом случае диаграмма вытянута вдоль оси размера пучка. Второй тип описывает узкий пучок с увеличенными углами наклона траекторий, и диаграмма вытянута вдоль оси углов. Пучок в этом случае сфокусирован в узкое пятно.

Площадь фазовой диаграммы называется эмиттансом пучка $\varepsilon$. Это сохраняющаяся величина, в то время как форма диаграммы зависит от характера фокусировки в электромагнитных полях. Эмиттансы пучка в точках с разными потенциалами подчиняются следующему соотношению:

$$
\varepsilon_{1} \sqrt{\varphi_{1}}=\varepsilon_{2} \sqrt{\varphi_{2}}
$$

Если контуры диаграмм в кроссовере представлены кривыми линиями, то это свидетельствует о нелинейных эффектах. Таким образом, фазовая диаграмма дает наглядное представление о качестве фокусировки. 


\section{1. Приведенные фазовые диаграммы}

Фазовая диаграмма расходящегося (или сходящегося) пучка выглядит как отрезок прямой линии. Уравнение этой линии равно $z^{\prime}=(\operatorname{tg} \alpha) z$. При этом угол наклона $\alpha$ гораздо больше углового разброса фазовых точек. По этой причине детали распределения фазовых точек в этих случаях не видны.

Рассмотрим сначала расходящийся пучок. В качестве примера на рис. 1 представлена диаграмма такого пучка. Для демонстрации специально выбран пучок с нелинейной фокусировкой. По виду диаграммы можно лишь сказать, что пучок расходится, не более того. Чтобы судить о качестве фокусировки, эту диаграмму следует привести к диаграмме кроссовера. Поскольку существует два типа кроссовера, то имеется два способа приведения. Для кроссовера первого типа сохраняется величина $z$, а угол наклона $z^{\prime}$ уменьшается на величину $z \operatorname{tg} \alpha_{0}$, где $\operatorname{tg} \alpha_{0}=\bar{z}^{\prime} / z$, а среднее значение угла вычисляется при заданном размере $z$. Эта процедура соответствует преобразованию фазовых координат с помощью фокусирующей тонкой линзы. Соответствующее матричное произведение имеет следующий вид:

$$
\left(\begin{array}{l}
x \\
x^{\prime}
\end{array}\right)_{\text {add }}=\left(\begin{array}{cc}
1 & 0 \\
-\operatorname{tg} \alpha_{0} & 1
\end{array}\right)\left(\begin{array}{l}
x \\
x^{\prime}
\end{array}\right) .
$$

При втором способе приведения сохраняются углы наклона, но уменьшается размер. Кроссовер второго типа достигается при движении частиц в обратную сторону на расстояние $l=\operatorname{ctg} \alpha_{0}$. Соответствующая матрица свободного промежутка имеет следующий вид:

$$
\left(\begin{array}{cc}
1 & -\operatorname{ctg} \alpha \\
0 & 1
\end{array}\right)
$$

Две приведенные фазовые диаграммы для расходящегося пучка, представленного на рис. 1, изображены на рис. 2. Диаграммы указывают на нелинейные эффекты при прохождении пучка в оптической системе. Из диаграмм следует, что в линзе, с помощью которой сформирован расходящийся пучок, начиная с расстояния от оси

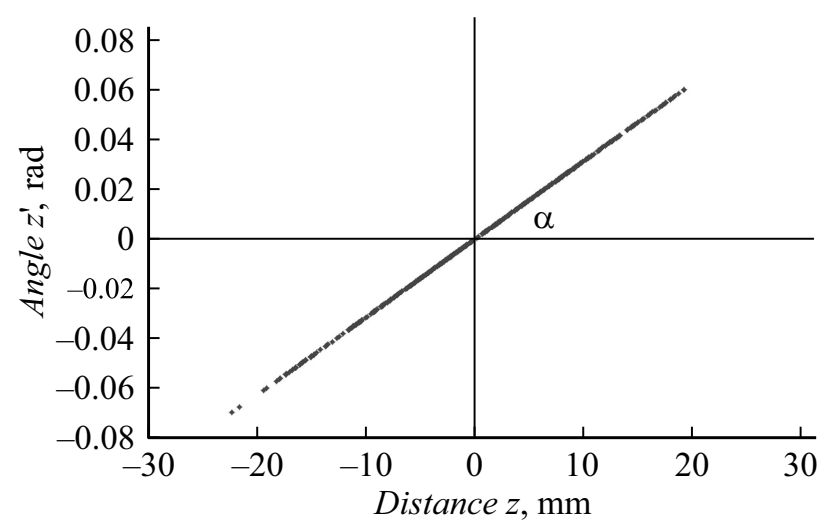

Рис. 1. Фазовая диаграмма расходящегося пучка.
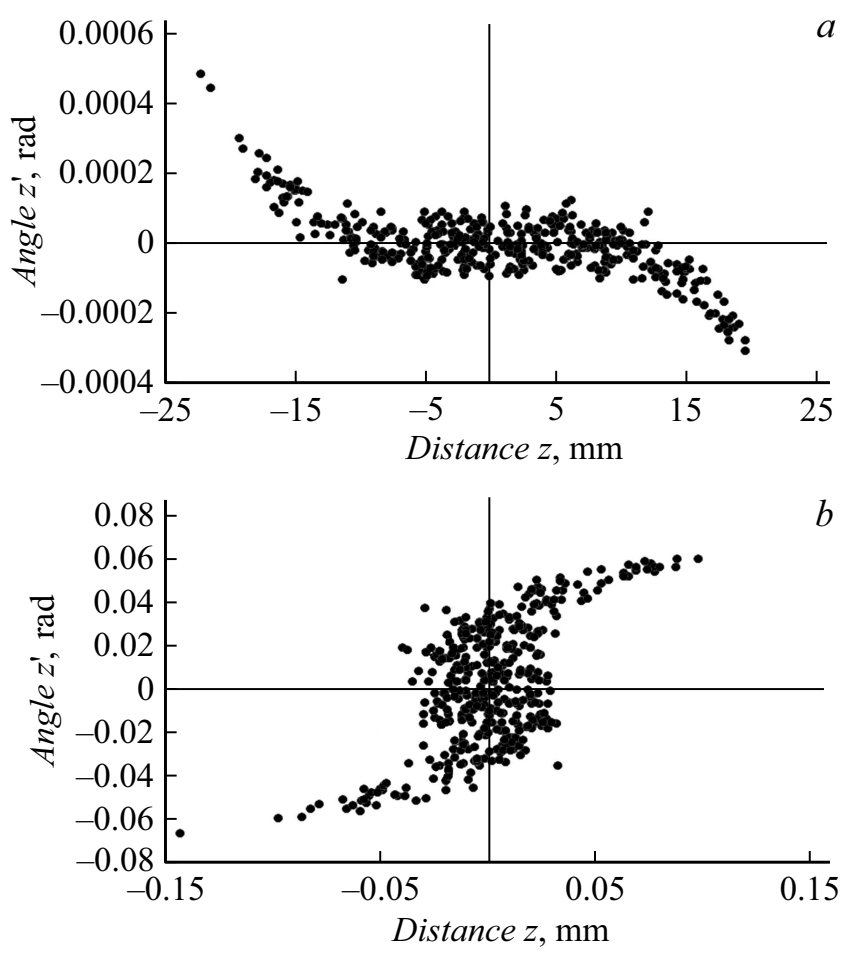

Рис. 2. Диаграммы пучка в кроссоверах: $a$ - диаграмма, полученная с помощью фокусирующей матрицы, $b$ - диаграмма, полученная с помощью матрицы свободного промежутка.

в $10 \mathrm{~mm}$ и более следует усилить оптическую силу. Иначе говоря, в этой области пространства электрическое поле недостаточно для линейной фокусировки.

Для сходящегося пучка угол $\alpha_{0}$ отрицателен. Первая матрица в этом случае является матрицей рассеивающей линзы, а положительный свободный промежуток указывает положение кроссовера при движении частиц вперед.

Таким образом, техника фазовых диаграмм позволяет в любом сечении трассы пучка измерить фазовую диаграмму и тестировать оптическую систему на наличие нелинейных эффектов. А это, в свою очередь, открывает возможность спроектировать корректоры аберраций или разработать линзы с линейной фокусировкой.

\section{2. Компьютерное моделирование}

Моделирование сводится к двум этапам. На первом этапе вычисляются распределение электростатических потенциалов для заданного расположения и геометрии электродов, градиенты потенциалов во внутренних областях электродов и межэлектродном пространстве, распределение магнитных полей в межполюсных зазорах магнитов и рассеянных полей в пространстве вне полюсов.

Распределение электростатических потенциалов описывается уравнением Лапласа, и геометрия электродов задает начальные условия для решения этого уравнения. Решение ищется методом итераций на объемной сетке. 
Магнитное поле определяется как циркуляция векторного потенциала, который зависит от токов в катушках магнита, их однородности, от величины и распределения магнитной проницаемости в магнитопроводе и материале полюсных наконечников и т.д. Поэтому нахождение распределения магнитного поля через векторный потенциал представляется очень трудной задачей и используется альтернативная возможность. Поскольку в пространстве вне полюсов отсутствуют заряды и токи, магнитное поле может рассматриваться как потенциальное $(\operatorname{rot} \mathbf{H}=0)$. В таком представлении вводится скалярный магнитный потенциал, хотя, как известно, магнитных зарядов не существует в отличие от скалярного электростатического потенциала, определяемого электрическими зарядами. Затем также как и для электростатической оптики решается уравнение Лапласа.

На втором этапе методом Рунге-Кутта производится расчет ионных траекторий от источника до детектора. Известный ход траекторий позволяет в любом заданном сечении пучка определить любые параметры частицы: отклонение от оси, массы, углы наклона траектории, энергии и скорости, времена пролета, фазовые диаграммы и ряд других параметров. При таких вычислениях предполагается, что ионные токи малы и не влияют на распределения полей, т.е. пренебрегается влиянием пространственного заряда. Пренебрегается также рассеиванием частиц на остаточном газе.

При вычислениях использовался программный продукт Simion 8 [6], пакет SolidWorks, созданные авторами программы, описывающие расположение и геометрию ионно-оптических элементов, а также стандартный пакет Microsoft Office Excel 2010 со встроенным пакетом Microsoft Visual Basic. Используемая оперативная память равнялась $16 \mathrm{~Gb}$.

\section{3. Источник ионов}

Для тестирования различных ионно-оптических элементов использовался типичный для масс-сепараторов пучок, извлекаемый из источника электрическим полем,

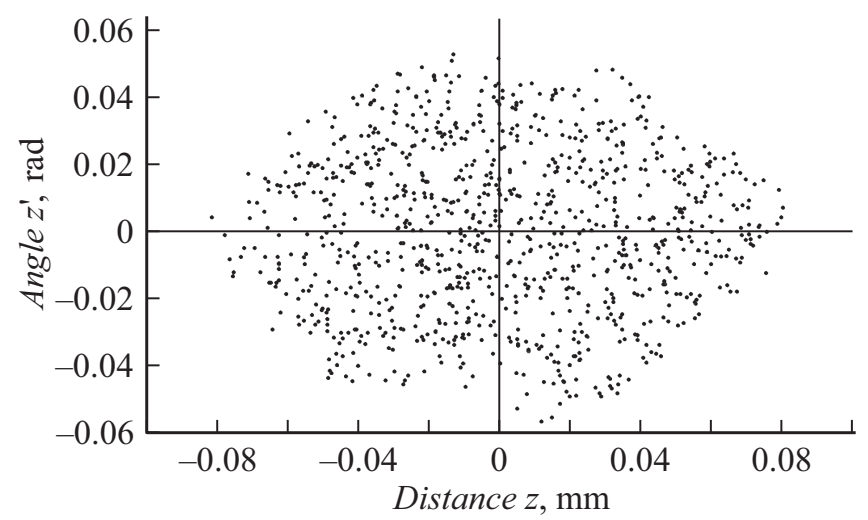

Рис. 3. Диаграмма пучка на выходе из источника.

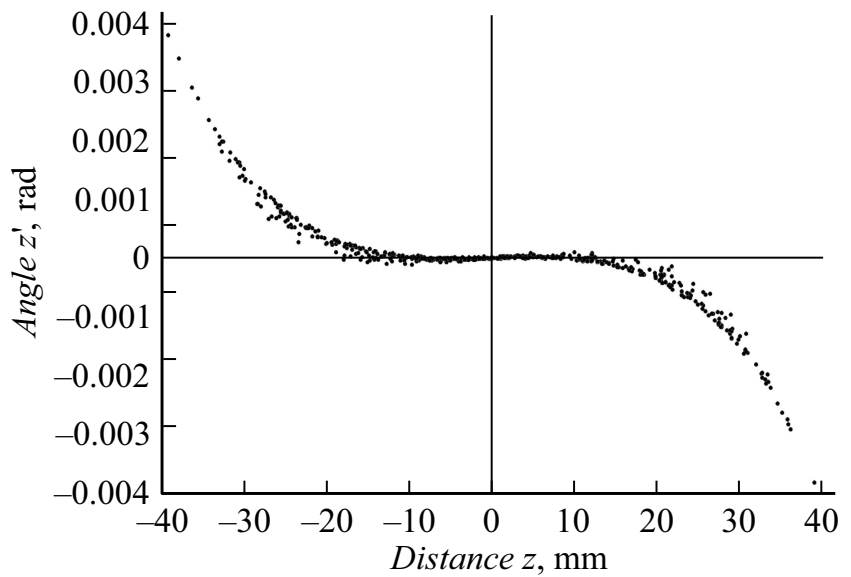

Рис. 4. Фазовая диаграмма пучка ионов в горизонтальной плоскости (плоскость $z, x$ ).

создаваемым вытягивающим электродом. Поле проникает в источник через отверстие диаметром $1 \mathrm{~mm}$, захватывает ионы и ускоряет их. Вблизи торцевой грани в точке с потенциалом $1 \mathrm{kV}$ имеется кроссовер пучка. Фазовая диаграмма в этой точке представлена на рис. 3. Контур диаграммы представляет собой ромб, что указывает на отсутствие нелинейных эффектов.

При ускорении частиц до энергии $30 \mathrm{keV}$ эмиттанс пучка уменьшается в соответствии с выражением (2) и принимает значение $4 \mathrm{~mm} \cdot \operatorname{mrad}$. Это типичное значение для ряда масс-сепараторов.

С таким ионным источником рассмотрим фокусировку частиц в квадрупольных, аксиально симметричных и трансаксиальных линзах. Первые два типа линз используются в ряде масс-сепараторов, работающих в различных научных центрах.

\section{4. Оптические свойства электростатических линз и секторных элементов}

\section{1. Квадрупольный триплет}

Квадрупольный триплет имеет следующие размеры: диаметр апертуры триплета равен $100 \mathrm{~mm}$, а диаметр электродов $118 \mathrm{~mm}$. Соответствующие длины электродов равны 125, 250 и $125 \mathrm{~mm}$. Вытягивающий электрод ионного источника находится под нулевым потенциалом, поэтому энергия ионов перед триплетом равна $30 \mathrm{keV}$. Потенциалы на электродах квадруполей равны \pm 1700 , $\mathrm{m} l 1700, \pm 1535 \mathrm{~V}$ соответственно. Пучок ионов на выходе триплета приблизительно параллелен. Его фазовая диаграмма в горизонтальной плоскости представлена на рис. 4. В этой плоскости выбран режим фокусировки триплета ФДФ. Здесь Ф означает „фокусировка“, а Д - „дефокусировка“. Пучок в этом случае в два раза шире, чем его размер в вертикальной плоскости, где фокусировка ДФД. 

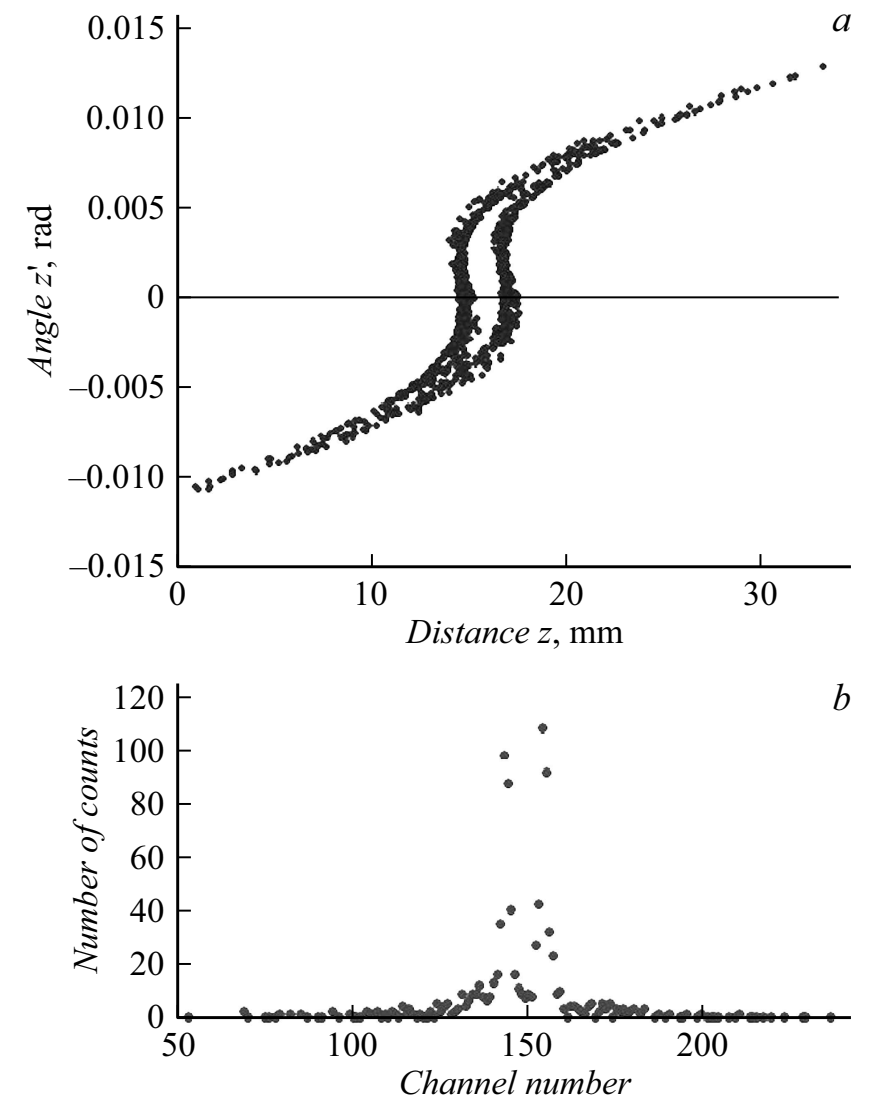

Рис. 5. $a-$ фазовые диаграммы пуска ионов с массами 100 и 100.1 в фокальной плоскости магнита, $b-$ массовый спектр.

Как видно из рис. 4, нелинейность начинает проявляться для траекторий, отстоящих от оси пучка более чем на $\pm 15 \mathrm{~mm}$, а углы наклона крайних траекторий пучка примерно в 20 раз больше углов в линейной области.

Рассмотри теперь свойства масс-сепаратора с квадрупольным триплетом. Секторный магнит сепаратора имеет радиус кривизны траектории, равный $1500 \mathrm{~mm}$, угол поворота $54.7^{\circ}$ и угол сектора $19.2^{\circ}$. Такой магнит описан в работе [7].

На рис. 5, а изображены фазовые диаграммы пучка в фокальной плоскости магнита. Пучок состоит из ионов, имеющих массы 100 и 100.1. Линейная часть фазовой диаграммы рис. 4 отображается в вертикальные участки фазовых диаграмм в фокальной плоскости магнита (рис. 5,a), а нелинейные участки отображаются на полностью перекрывающиеся нелинейные участки диаграмм. При свертке диаграмм по углу в фокальной плоскости получается спектр масс. Этот спектр изображен на рис. $5, b$.

Нелинейность фокусировки в квадрупольном триплете приводит к тому, что линии каждой массы содержат примеси соседней массы. Почти полное перекрытие масс характерно для склонов линий. При ширине пиков на половине высоты, равной двум каналам, каждая линия по основанию занимает примерно 130 каналов. Это отрицательное свойство в сепарации масс можно устранить двумя способами. Первый заключается в установке на выходе триплета коллиматора, который не пропускает ионы с нелинейной фокусировкой. Ширина коллиматора составляет 30-40 mm. Но в этом случае частично теряется эффективность сепаратора. При втором более предпочтительном способе используется корректор аберраций, который в системе триплет-корректор позволяет получить линейную фазовую диаграмму.

\section{2. Аксиально-симметричная линза}

Фазовая диаграмма пучка на выходе трехэлектродной аксиально-симметричной линзы с апертурой $100 \mathrm{~mm}$ похожа на рис. 4, с той лишь разницей, что нелинейность начинает проявляться при отклонении от оси на расстояние, превышающее величину $\pm 5 \mathrm{~mm}$. Область линейной фокусировки у этой линзы меньше, чем у квадрупольной линзы. По этой причине масс-сепараторы с аксиальными линзами имеют невысокую разрешающую способность, в пределах 500-1000 [5].

\section{3. Трансаксиальная линза}

Трансаксиальные линзы и их свойства описаны в работах [8-10]. Несмотря на ряд положительных свойств, они не получили распространения в мировом приборостроении.

В горизонтальной плоскости фокусные расстояния линзы зависят от радиусов кривизны зазоров между электродами и потенциалов на электродах. Если частица в области зазора ускоряется, то при движении в направлении центра кривизны частица фокусируется, а при движении от центра кривизны - дефокусируется. В вертикальной плоскости такие линзы всегда фокусируют.

Один из возможных простых вариантов геометрии такой линзы представлен на рис. 6. Параметры подобраны таким образом, что пучок частиц приблизительно параллелен в обеих плоскостях.

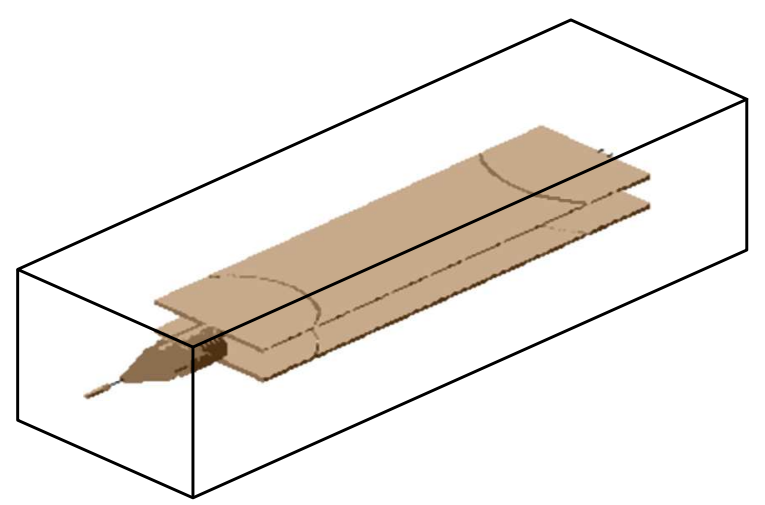

Рис. 6. Трансаксиальная линза с ионным источником и вытягивающим электродом. 

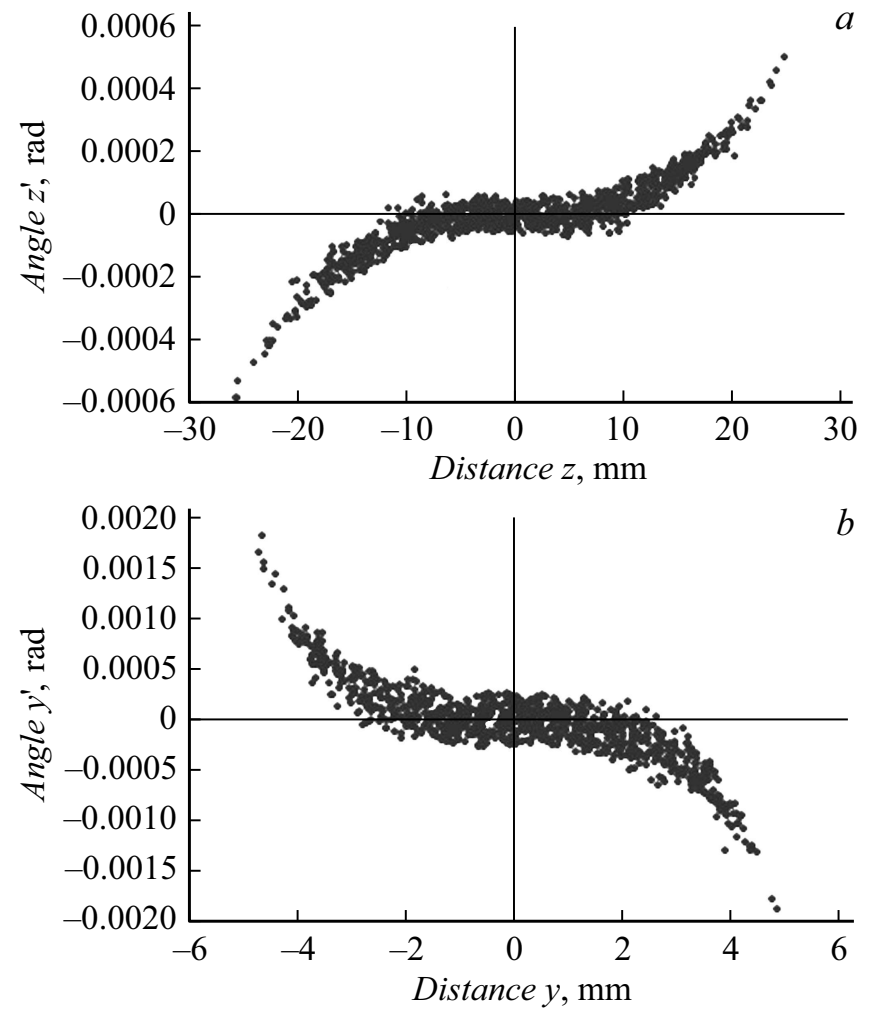

Рис. 7. Фазовые диаграммы пучка на выходе трансаксиальной линзы: $a-$ в горизонтальной плоскости, $b-$ в вертикальной плоскости.

Приведем геометрические параметры моделируемой система источник-линза. Источник - это трубка с внутренним диаметром, равным $4 \mathrm{~mm}$, и торцевым отверстием $1 \mathrm{~mm}$. Вытягивающий электрод, соединенный с первым электродом линзы, имеет длину $120 \mathrm{~mm}$ и выходную апертуру $40 \mathrm{~mm}$. Зазор между пластинами электродов равен $40 \mathrm{~mm}$, а длины электродов равны 165, 406 и $110 \mathrm{~mm}$ соответственно. Плоскопараллельные пары электродов в этой линзе разделены круговыми зазорами со средними значениями радиусов, равными $172.5 \mathrm{~mm}$ для первого зазора и $252.5 \mathrm{~mm}$ для второго. Потенциалы, начиная с источника, последовательно равны $30,18,10.6$ и $0 \mathrm{kV}$.

В области первого зазора линзы частицы рассеиваются, а в области второго пучок ионов фокусируется. Фазовые диаграммы пучка на выходе линзы представлены на рис. 7.

В отличие от аксиальной и квадрупольных линз в данном случае характер нелинейности в фокусировке иной. С ростом отклонения траектории от оси в горизонтальной плоскости наблюдается недостаток в величине электростатического поля и внешние траектории пучка расходятся.

К достоинству линзы относится то, что она формирует ленточный пучок. Ширина его в горизонтальной плоскости равна $50 \mathrm{~mm}$. В вертикальной плоскости ширина пучка равна $9 \mathrm{~mm}$, крайние траектории при этом сходятся, а область линейной фокусировки составляет $4 \mathrm{~mm}$.

\section{4. Секторный магнит}

На рис. 8 представлены фазовые диаграммы пучков ионов в фокальной плоскости рассмотренного ранее секторного магнита масс-сепаратора. Чтобы отобразить фазовые диаграммы на одном рисунке в зависимости от ширины входного пучка, массы ионов сдвинуты на величину 0.025 .

Как видно из рисунка, ширина фазовой полоски уменьшается пропорционально входному размеру пучка, и пропорционально этому размеру увеличиваются аберрации. Если устранить эти аберрации, то разрешающая способность сепаратора с входным пучком $80 \mathrm{~mm}$ была бы в четыре раза выше, чем у сепаратора, имеющего входной пучок шириной $20 \mathrm{~mm}$. Для увеличения разрешающей способности магнит нуждается в корректоре аберраций, если ширина пучка на входе магнита больше, чем $40 \mathrm{~mm}$.

\section{5. Цилиндрический конденсатор}

Фазовая диаграмма пучка на выходе цилиндрического конденсатора имеет серпообразный вид, похожий на диаграмму рис. 8 для массы 100.05 . Секторный магнит и цилиндрический конденсатор демонстрируют новый вид нелинейных искажений фокусировки, отличающийся симметрией от нелинейных искажений в электростатических фокусирующих линзах. Левые части этих диаграмм указывают как на сходящиеся (второй квадрант) так и на расходящиеся (третий квадрант) траектории. Это общее свойство секторных отклоняющих систем.

Частицы, летящие по внешним относительно центра кривизны траекториям пучка, пересекают осевую линию перед фокальной плоскостью магнита (или цилиндрического конденсатора) и фазовые координаты таких

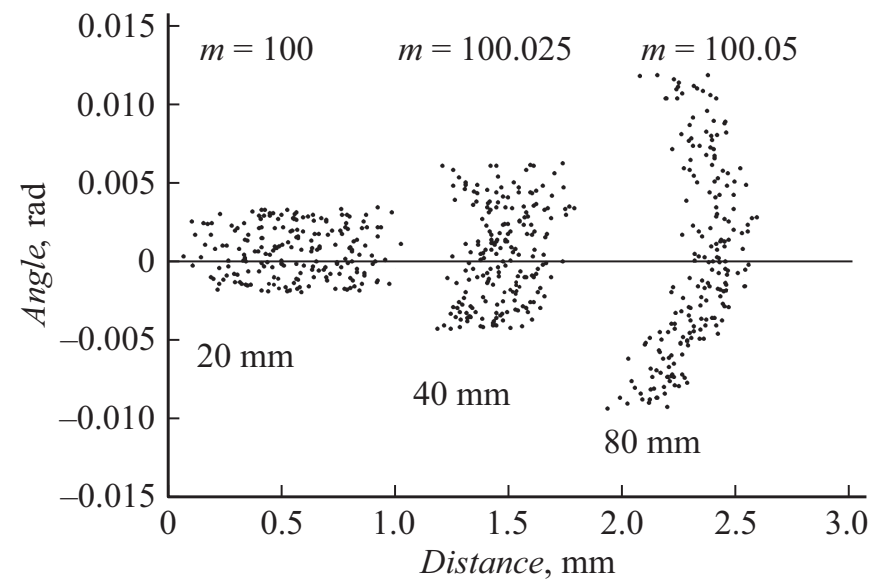

Рис. 8. Фазовые диаграммы в фокальной плоскости магнита. Входной пучок содержит массы 100, 100.025 и 100.05 с входными ширинами 20,40 и $80 \mathrm{~mm}$ соответственно. Каждый из трех пучков имеет эмиттанс $4 \mathrm{~mm} \cdot \mathrm{mrad}$. 
частиц находятся в третьем квадранте. Внутренние же траектории пересекают осевую линию за фокальной плоскостью, поэтому на фазовой диаграмме эти сходящиеся траектории отображаются во втором квадранте.

\section{Заключение}

Показано, что метод фазовых диаграмм позволяет наглядно определить качество фокусировки различными ионно-оптическими элементами. В квадрупольных и аксиально-симметричных линзах нелинейность появляется в областях, где значение электростатического поля больше, чем величина поля, необходимая для линейной фокусировки. В трансаксиальных линзах знак нелинейности противоположен. Искажение линейности обусловлено недостаточной величиной поля, и для сохранения линейности это поле следует увеличить. Такая связь аберраций с фокусирующим полем дает возможность рассчитать и сконструировать корректоры, использование которых в системе линза-корректор приводит к линейным фазовым диаграммам.

С другой стороны, модификация плоскопараллельных электродов трансаксиальной линзы позволяет реализовать линейную фокусировку ионов. Геометрия и свойства линейно фокусирующей линзы, а также корректоров аберраций для нелинейно фокусирующих линз и секторных магнитов будут рассмотрены в следующей работе.

\section{Конфликт интересов}

Автор заявляет, что у него нет конфликта интересов.

\section{Список литературы}

[1] Лоусон Джс. Физика пучков заряженных частиц. М.: Мир, 1980. 439 c. [Lawson J.D. The Physics of Charged-Particle Beams. Oxford: Clarendon Press, 1977. 462 p.]

[2] Бенфорд А. Транспортировка пучков заряженных частиц. М.: Атомиздат, 1969. 240 с. [Banford A.P. The Transport of Charged-Particle Beams. London: Spon, 1966. 229 p.]

[3] Вольник Г. Оптика заряженных частиц. СПб.: Энергоатомиздат, 1992. 280 c. [Wollnik H. Optics of charged particles. Academic Press, 1987. 304 p.]

[4] Panteleev V.N. et al. Main scientific activities. Gatchina: PNPI, HEPD, 2013. P. 274.

[5] Lawson J.D. et al. // Particle Acceler. 1973. Vol. 5. P. 61.

[6] Электронный ресурс. Описание программы Simion. Version 8.1. Режим доступа: http: // www.simion.com

[7] Rudstam G. // Nucl. Instrum. Meth. 1976. Vol. 139. P. 239.

[8] Карецкая С.П., Кельман В.М., Якушев Е.М. // ЖТФ. 1970. T. 40. C. 2563.

[9] Карецкая С.П., Кельман В.М., Якушев Е.М. // ЖТФ. 1971. T. 41. C. 325.

[10] Карецкая С.П., Кельман В.М., Якушев Е.М. // ЖТФ. 1971. T. 41. C. 548. 